\title{
Las ideas de los padres sobre sus hijos en la investigación evolutiva*
}

\section{Jesús Palacios \\ Universidad de Sevilla**}

\section{EMERGENCIA DE LOS PROCESOS COGNITIVOS DE LOS PADRES EN LA INVESTIGACION EVOLUTIVA}

Cada vez que alguien se pregunta por qué los niños evolucionan y se comportan como lo hacen, la referencia a los padres es uno de los elementos de obligada presencia en la respuesta. En concreto, los psicólogos evolutivos interesados en el estudio del desarrollo infantil no han tenido por menos que volver la vista a los padres cada vez que se han preguntado por los determinantes del comportamiento del niño. Con independencia del punto de vista teórico que cada uno adopte, ningún psicólogo infantil pone en duda el importante papel que la familia juega en la configuración del ser psicológico del niño. No debe extrañar, por tanto, que el estudio de la relación entre las características de la familia y las del niño sea uno de los temas clásicos de la Psicología Evolutiva.

Históricamente se han producido importantes cambios en la forma de llevar a cabo tal estudio, cambios que reflejan mutaciones que afectan tanto a los enfoques teóricos predominantes, que han ido sufriendo un largo e interesante proceso de sustituciones, cuanto a la metodología de estudio y de análisis de los datos, ámbito éste en el que los cambios con el paso del tiempo han sido también habiruales. De esta forma, unos enfoques han ido cediendo terreno a otros y nuevos temas de investigación han ocupado el espacio otrora dominado por temas diferentes.

Uno de los dominios de aparición reciente en la palestra de las investigaciones actuales sobre esta temática tiene que ver con un conjunto de hechos psicológicos que bien pudiera agruparse bajo el encabezamiento general de «procesos cognitivos de los padresw. Poco a poco se ha ido haciendo evidente que las conductas de los padres no son respuestas ni aleatorias ni automáticas a las conductas del niño (McGillicuddy-DeLisi, 1982)***, que los padres ni son una ta-

* Una versión mucho más extensa de este trabajo y el que le sigue se puede encontrar en Palacios (en prensa).

** Dirección del autor: Departamento de Psicología Evolutiva y de la Educación. Universidad de Sevilla. Apartado 3128. 41071 SEVILLA.

*** Las referencias bibliográficas de este artículo se encuentran al final del artículo siguiente de este dossier. 
bula rasa respecto al desarrollo y la educación de los niños cuando reciben la llegada de su primer hijo (Sutherland, 1983), ni actúan esin mentes ante el niño, como si se limitasen sólo a reaccionar a su última conducta o a registrar pasivamente lo que el niño hace (Goodnow, 1981). Se ha ido así abriendo paso un área de estudio que intenta explorar lo que los padres piensan sobre sus hijos, sobre su desarrollo y educación, lo que esperan de ellos, a qué atribuyen su comportamiento y las ideas que tienen respecto a cómo influir sobre él, etc. Si se considera que la conducta a que se despliega tiene, entre otros, un enraizamiento cognitivo y que los ámbitos del conocimiento y la conducta de una persona no están disociados entre sí, no es de extrañar que los investigadores hayan querido explorar primeramente los contenidos de los procesos cognitivos de los padres referidos al desarrollo y educación de los niños para, en un segundo término, explorar las relaciones entre dichos procesos cognitivos y las conductas educativas de los padres en relación con sus hijos.

Como algunos autores han señalado (Goodnow, 1981), es en cierto modo sorprendente que este tema haya suscitado hasta no hace mucho un escaso interés, pues las ideas en torno a los niños y su educación forman parte del conocimiento popular y encuentran expresión en el folklore, las tradiciones, el refranero, etc. (Sutherland, 1983). Hay que señalar, no obstante, que al menos la constatación de la importancia del tema no es de última hora. Ya en una revisión de literatura sobre la influencia de los padres en el desarrollo cognitivo de los niños pequeños, Freeberg y Payne (1967) constataban la existencia de un vacío en la investigación sobre los procesos cognitivos de los padres en torno a la crianza y estimulación de sus hijos. Poco tiempo después, en una monografía de Emmerich (1969), el rol de padres es visto como sun rico tejido de procesos cognitivos interrelacionados» (p. 43). En el contexto de trabajos más generales, tanto Baldwin (1967) como Wegner y Vallacher (1977) se refirieron a la «psicología ingenuas o «psicología implícitas de los padres en torno a los niños, su desarrollo y educación.

A pesar de que estos trabajos supusieron un cierto impulso para el área de investigación al que nos estamos refiriendo, y a pesar de que a uno y otro lado del Atlántico se produjeron excelentes publicaciones sobre estos temas (Boltanski, 1969; Broussard y Hartner, 1971; Kohn, 1969; Newson y Newson, 1963, 1968, 1976; Whiting, 1974, por citar algunos ejemplos), a finales de la década de los 70 tanto Parke (1978) como Bell (1979) constataban la escasez de investigaciones empíricas y alentaban a una profundización en el estudio de los procesos cognitivos de los padres referidos al desarrollo y educación de sus hijos.

Por fortuna, la temática ha irrumpido con fuerza en la década de los 80 , propiciando no sólo investigaciones empíricas, sino también modelos teóricos en los que encuadrar los datos de ellas derivados. Aunque desde luego no se limite a ellos, el tema ha encontrado arraigo y desarrollo en dos núcleos académicos principales: el Educational Testing Service, en Princeton (Estados Unidos), y la Universidad Macquarie, en Sydney (Australia); al primero están asociados los nombres de McGillicuddy-DeLisi y de Sigel; al segundo, el de Goodnow. De las aportaciones de todos ellos y de otros muchos investigadores dan fe tanto las referencias que aparecen al final del siguiente artículo de este dossier como la apretada revisión de literatura de las páginas que siguen. En Sigel (1985a) y en Ashmore y Brodzinski (1986), se encuentran abundantes muestras del estado actual de la cuestión tanto a nivel empírico como conceptual. En España, Palacios (1984, 1985a, y b) en la Universidad de Sevilla, y Rodrigo (1985; Triana y Rodrigo, 1985), en la de La Laguna, se han ocupado de estas cuestiones. Por lo demás, la temática de las ideas de los padres no sólo se ha afincado en la investigación evolutiva, sino que además, como ha señalado Goodnow (1987), 
a pesar de ser éste un campo inicialmente vinculado a los planteamientos cognitivo-evolutivos, en los últimos años ha ido estableciendo conexiones con planteamientos más típicos de la psicología cognitiva y de la psicología social. Lamentablemente, no disponemos aquí de espacio para entrar en los detalles de estas conexiones ni para abordar la temática, relacionada en buena parte con ellas, del cambio de las ideas de los padres. Baste citar, como muestra, el mencionado trabajo de Goodnow (1987) y los de Cantor y Kihlstrom (1987), Crocker, Fiske y Taylor (1984) y Moscovici (1984).

Se extrañaba Parke (1978) de que a pesar del revival cognitivo de las décadas del 60 y el 70 en el ámbito de la Psicología, los procesos cognitivos de los padres en torno al desarrollo y la educación de sus hijos hubiesesn suscitado tan escaso interés y hubieran propiciado tan pocas investigaciones. Según lo entendemos nosotros, la primacía de lo cognitivo en los intereses de los investigadores era condición necesaria pero no suficiente para dar lugar al tipo de investigaciones que Parke (1978) echaba con razón en falta. Era preciso no sólo que plantearse el problema fuera posible (posibilidad que venía dada por el auge del cognitivismo), sino que los investigadores lo sintieran además como nęcesario. Tal necesidad se ha ido haciendo patente, según creemos, a medida que el estudio de los procesos de interacción padres-hijo ha ido convirtiéndose en preocupación dominante entre los psicólogos evolutivos.

Gracias a la investigación en el área de los procesos interactivos, hemos ido conociendo lo que el bebé aporta a la interacción y las capacidades cognitivas que están detrás de esa aportación. En el intento de conocer lo que los padres, por su parte, aportan, lógico es que los investigadores hayan sentido la necesidad de adentrarse en los procesos y contenidos cognitivos que, junto a otros contenidos y procesos, están implícitos tras la conducta que los padres desarrollan en sus interacciones con el niño. Así, a juicio de McGillicuddy-DeLisi (1985), el interés reciente por esta temática se basa en el supuesto según el cual è origen de la variabilidad entre unos padres y otros, así como la estructura subyacente que da coherencia a la conducta de un padre determinado observado en distintos momentos y contextos, pueden encontrarse formando un conjunto coherente y comprensible de ideas que, en consecuencia, se hace necesario estudiar.

\section{DIVERSIDAD Y ESTRUCTURA DE LAS IDEAS DE LOS PADRES}

Uno de los problemas que plantea el área de trabajo que nos ocupa radica en la diversidad de contenidos a que se refiere. Cuando hablamos de los procesos cognitivos de los padres en torno al desarrollo y educación de sus hijos, estamos englobando en esa expresión genérica las creencias, las actitudes, los valores, las expectativas, las inferencias, las atribuciones, etc., que los padres tienen respecto a los niños. El inconveniente de una expresión genérica es, lógicamente, su ausencia de matices y las generalizaciones que alienta, generalizaciones que son tanto más indeseables cuanto menos fundadas estén. La expresión «procesos cognitivos de los padres» incluye contenidos tan diversos que cualquiera que sea la definición que de ella se dé, resultará parcial, vaga, imprecisa e insatisfactoria. Si englobamos bajo un mismo epígrafe conceptual, por ejemplo, lo que los padres saben sobre el desarrollo, las inferencias que hacen respecto al comportamiento del niño y las expectativas que tienen en relación con su futuro, difícilmente ocurrirá que conocimientos, inferencias y expectativas se hayan formado de la misma manera, tengan idéntica dinámica y se traduzcan de forma similar en la interacción cotidiana del padre con su hijo. Tal vez lo que sea 
verdad en el caso de los conocimientos sea sólo correcto a medias en el caso de las inferencias, mientras en el de las expectativas las cosas funcionen de otra manera.

Creemos por ello que con independencia de que exista o no una etiqueta genérica, los contenidos cognitivos que los padres poseen en torno al desarrollo y la educación de sus hijos deben ser conceptualizados de manera diversificada. Autores como Goodnow (1985), Parke (1978) y Bacon y Ashmore (1986), han hecho propuestas en este sentido, si bien consideramos que limitadas a sólo algunos de los procesos cognitivos de los padres sobre el desarrollo y la educación de sus hijos. De acuerdo con nuestra propia propuesta, sería necesario referirse a los siguientes procesos cognitivos de los padres: percepciones, conocimientos, suposiciones e inferencias, valores o aspiraciones, expectativas, actitudes y atribuciones; a estos siete contenidos se deben añadir la capacidad percibida de influencia que los padres creen tener sobre el desarrollo y la educación de sus hijos, y las estrategias educativas preferidas por aquéllos en su relación con éstos. A todos estos contenidos se deben agregar las siguientes cualidades: grado de coherencia interna entre diversos contenidos, grado de flexibilidad, carácter más o menos estereotipado, status más o menos implícito o explícito de los mencionados contenidos, y, por último, su carácter más o menos abstracto o concreto.

Aunque es evidente que los conocimientos y cualidades a que nos hemos referido no conforman entre sí realidades separadas ni compartimentos estancos, una conceptualización diversificada como la que proponemos refleja mejor la complejidad de lo real de lo que lo hacen términos genéricos.

Siendo preferible, pues, la diversificación, se ha de reconocer que resulta cómodo disponer de un término genérico, siempre y cuando se tenga conciencia de sus limitaciones y no se haga de él una utilización abusiva que conduzca a ignorar la diversidad o al intento de amalgamarla indebidamente. De los distintos términos genéricos posibles (procesos cognitivos, creencias, teorías implícitas...), hemos elegido la palabra «ideas» para referirnos a los contenidos y cualidades más arriba mencionados, y la expresión sideas evolutivo-educativas de los padres» para aludir abreviadamente al objeto de nuestro estudio.

Pensando aún en términos genéricos, es interesante mencionar la propuesta de Sigel (1985b) según la cual se puede hacer una distinción entre ideas referidas al qué e ideas relativas al cómo; las primeras son ideas de tipo frecuentemente general sobre de qué forma son los niños, de qué manera evolucionan, mientras que las segundas se refieren a la forma en que los padres creen que se debe actuar en determinadas situaciones o para lograr unos u otros objetivos. A la hora de establecer conexiones entre las ideas de los padres y sus comportamientos educativos, las ideas relativas al cómo permitirán, probablemente, hacer predicciones de conducta más ajustadas, lo que no significa, sin embargo, que las ideas relativas al qué carezcan de plasmación conductual, pues, por poner un solo ejemplo, las expectativas de rendimiento que un padre determinado tenga respecto a su hijo en un ámbito concreto, muy probablemente hallarán traducción en algún aspecto de su interacción educativa con él.

Pero de las ideas evolutivo-educativas de los padres nos interesa, a más de su diversidad, su estructura. Las opiniones respecto a la organización, interrelaciones y status de las ideas evolutivo-educativas de los padres no son, desde luego, unánimes. Para algunos autores, los padres tienen ideas claras respecto a la naturaleza de los niños y el desarrollo infantil (McGillicuddy-DeLisi, 1982b), ideas que se suponen interrelacionadas y ordenadas entre sí jerárquicamente (Sigel, 1985b) y que se cree forman un sistema organizado de conocimientos (Newberger, 1980). Quienes sostienen este punto de vista son además proclives 
evolutivos, afirmando que, aunque los padres no presenten sus ideas en la jerga y con la sistematización de la literatura psicológica, no obstante son reconocibles en ellas sistemas de creencias que recuerdan a enfoques maduracionistas, conductistas, constructivistas, etc. (McGillicuddy-DeLisi, Sigel y Johnson, 1979; Triana y Rodrigo, 1985).

En el extremo opuesto al anterior se sirúan posiciones que cuestionan la supuesta importancia de los procesos cognitivos de los padres. Así, por ejemplo, Langer (1978) ha sostenido que, como en el resto de las personas, en los padres existe un elevado porcentaje de actividad sin pensamientos, pues gran parte de la conducta humana se despliega de forma relativamente automática, ajustándose a scripts que con mucha frecuencia se han formado de manera acrítica, con escasa reflexión.

No es fácil compartir por completo ninguna de estas dos posiciones. De un lado, porque es bastante evidente que, aún aceptando la existencia de una importante proporción de conductas con escaso sustrato racional, ello no es óbice para afirmar que los padres piensan en sus hijos, en cómo son y cómo se los debe tratar; además, muchas de las prácticas educativas que se desarrollan sin analizarlas a fondo, poseen en sí mismas, implícitas en ellas, unas determinadas concepciones evolutivo-educativas. De otro lado, porque el acientífico intuitivo» tiene más de intuitivo que de científico: generaliza sobre pocos datos, halla conexiones causales donde no existen, justifica frecuentemente su conducta con razones contextuales al tiempo que atribuye la conducta de los demás a rasgos de personalidad estables, desconoce la fragilidad de sus teorías, etc. (Bates y Bayles, 1984; Dix y Grusec, 1985; Goodnow, Knight y Cashomore, 1986, Rodrigo, 1985; Ross, 1981; Wegner y Vallacher, 1977).

Nos parece razonable aceptar que una buena proporción de las conductas de los padres respecto a sus hijos ocurre «sin pensamiento», pero creemos que no es menos cierto que, por una parte, hay también una proporción apreciable de conducta sujeta a una elaboración más propositiva, y, por otra, que, incluso si tal no es el caso, muchas de las conductas que los padres realizan sin pensar demasiado en ellas contienen tras de sí, como ya hemos señalado, implícitos cargados de contenido (Boltanski, 1969); al fin y al cabo, una conducta no precisa ser necesariamente explícita o consciente para poseer una cierta racionalidad, como el hecho de que una persona no sea consciente de las reglas de la gramática no se opone a la gramaticalidad de su lenguaje.

Parece también razonable postular, finalmente, que las diferentes ideas evolutivo-educativas de los padres no están aisladas unas de otras, como antes hemos mencionado. No quiere esto decir que todas las ideas deban estar relacionadas e interconectadas entre sí, formando un todo indisoluble y coherente, sino que probablemente hay grupos o familias de ideas entre las cuales se dan lazos de relación o dependencia. Creemos en este sentido aceptable la idea de McGillicuddy-DeLisi (1982a) y Sigel (1986) según la cual las ideas de los padres pueden estar organizadas en términos de subsistemas más pequeños en mayor o menor grado interrelacionados en el interior de un sistema más global, más amplio. Entre unos subsistemas y otros no tiene por qué haber necesariamente correspondencia lógica, pudiendo incluso darse contradicciones no ya entre un subsistema y otro, sino incluso en el interior de un subsistema determinado (Schaefer y Edgerton, 1985).

\section{CONTENIDOS DE LAS IDEAS EVOLUTIVO-EDUCATIVAS DE LOS PADRES}

Las investigaciones empíricas, cuyos resultados aquí tratamos de compendiar muy sumariamente, se han centrado más en unos aspectos que en otros y han 
dado lugar a un cierto nivel de consenso en unas áreas y de desacuerdo en otras. Los distintos contenidos abordados en dichas investigaciones empíricas nos parece que pueden agruparse bajo los cinco epígrafes siguentes: ideas sobre las causas de la conducta y los factores que la influyen; ideas sobre el calendario evolutivo; ideas sobre valores, expectativas, actitudes; ideas sobre cómo aprenden los niños y cuál es el papel de los padres; ideas sobre estrategias educativas. Estos cinco bloques de contenido agrupan en su interior los elementos más importantes de la diversidad de contenidos y cualidades a que nos referimos más arriba, sirviéndonos además para sistematizar las aportaciones contenidas en la literatura.

Por lo demás, los contenidos de estos cinco bloques ponen de manifiesto que hay terrenos en los que los investigadores no se han adentrado o lo han hecho muy escasamente. Así, por ejemplo, ocurre con las cuestiones que tienen que ver con el dominio de lo afectivo. En general, las ideas de los padres han sido más exploradas en el ámbito del desarrollo cognitivo y del aprendizaje, que en el ámbito de lo emocional. Recientemente, sin embargo, algunos autores han empezado a interesarse por este ámbito (Zelko, Duncan, Barden, Garber y Masters, 1986), respecto al cual Goodnow $(1984,1987)$ ha reivindicado un papel importante.

\subsection{Ideas sobre las causas de la conducta y los factores que la influyen}

De acuerdo con los datos de Schaefer y Edgerton (1985), las variables demográficas nivel educativo, ingresos familiares y pertenencia al grupo étnico mayoritario, correlacionan negativamente con ideas educativas de tipo tradicional, entre las que se incluye, por ejemplo, la creencia en que los niños son básicamente buenos o malos. Frankel y Roer-Bornstein (1982), por su parte, al indagar a qué atribuyen las madres los problemas que pueden surgir en el embarazo, encuentran también una correlación negativa entre la modernización cultural y/o generacional, por un lado, y la aceptación de explicaciones de carácter mágico-espiritualista, como el mal de ojo, por otro. Por poner sólo otro ejemplo, Boltanski (1969) afirma que la creencia en la influencia negativa de los antojos durante el embarazo, presenta una estrecha dependencia de los niveles de status más bajos.

Investigaciones preocupadas por contenidos distintos de los mencionados en el párrafo precedente, aunque complementarios a ellos, han puesto de manifiesto que los padres varían unos de otros en el grado en que creen que podrán influir sobre las cualidades psicológicas (cognitivas y no cognitivas) de sus hijos: los de más elevado status socioeconómico creen que pueden influir en gran medida, mientras que los de status bajo se inclinan más por la idea de que la edad del niño y sus características heredadas establecen límites sobre los que nada se puede hacer (Ninio, 1979; Zukow, en este dossier) (ver, más abajo, el apartado 3.4.).

Parece, pues, claro que hay padres que sostienen ideas de tipo innatistamaduracionista, mientras que otros están más próximos a planteamientos ambientalistas-interaccionistas. La dependencia de estas diferentes ideas respecto a variables de naturaleza sociocultural ya ha sido señalada, pero volveremos sobre ella más abajo.

\subsection{Ideas sobre el calendario evolutivo}

Al preguntar a los padres a qué edad creen que el niño adquiere o presenta una determinada destreza (ver, hablar, etc.), estamos indagando lo que unos 
han llamado calendario evolutivo (Goodnow, Cashmore, Cotton y Knight, 1984; Ninio, 1979) y aquello a lo que otros han caracterizado como la versión privada de las normas de Gessell sobre lo que es posible y deseable en cada momento del desarrollo (Hess, Kashigawa, Azuma, Price y Dickson, 1980).

Tanto Hess el al. (1980) como Ninio (1979) encuentran que los padres de nivel socioeconómico más elevado formulan predicciones evolutivas que implican mayor precocidad que la defendida por los de nivel socioeconómico bajo. Por su parte, Goodnow et al. (1984) no hallan diferencias respecto al calendario evolutivo en función de criterios de status, aunque sí encuentran tales diferencias al controlar la procedencia étnica y cultural de los padres estudiados. Las diferencias en función de la procedencia cultural han sido halladas también por Frankel y Roer-Bornstein (1982), así como por los citados Hess et al. (1980) y Ninio (1979).

Tanto Frankel y Roer-Bornstein (1982) como Ho y Kang (1984) han hallado en sus investigaciones que los padres jóvenes tienen una previsión de calendario evolutivo más precoz que la de sus propios padres, lo que muestra la incidencia del factor generacional sobre el tema que nos ocupa. La única limitación a esta generalización parece encontrarse entre las madres adolescentes, cuyas expectativas de calendario evolutivo son con frecuencia más pesimistas que las de sus propias madres (Fry, 1986; Stevens, 1984; Yong, 1981).

\subsection{Ideas sobre valores, expectativas, actitudes}

De acuerdo con Le Vine (1974), existen tres valores que tienen el carácter de metas universales que los padres sostienen respecto a sus hijos: el primero, la supervivencia física y la salud del niño; el segundo, el desarrollo de la capacidad conductual necesaria para que el niño pueda llegar a convertirse en un adulto económicamente independiente; el tercero, el desarrollo de capacidades conductuales que enfatizan otros valores (morales, emocionales, intelectuales...) típicos ya de cada cultura. Según Le Vine (1974) hay en esta tríada una jerarquía natural, de modo que cuando el primero de ellos se ve amenazado, se convierte en el más importante; lo mismo ocurre cuando, asegurado el primero, peligra el segundo. Esto significa que los padres valoran para sus hijos no sólo lo que a ellos les parece más importante en abstracto, sino también lo que más les preocupa en concreto, como han mostrado, entre otros, Burns, Homel y Goodnow (1984), Goodnow (1981, 1985), Lautrey (1980) y Lawton, Schuler, Fowell y Madsen (1984).

Por otra parte, las investigaciones de los últimos años están confirmando la tendencia que Kohn $(1969,1976$; Kohn y Schooler, 1969) describió hace tiempo y según la cual los valores relacionados con la autonomía, la independencia y la afirmación personal son preferidos para sus hijos por los padres de nivel socioeconómico elevado, mientras que los de status bajo enfatizan más valores de conformidad, obediencia y sometimiento a las normas (Alwin, 1984; Burns et al., 1984; Gerris, Vermulst, Franken y Janssens, 1986; Johnson y Martin, 1985; Lautrey, 1980; Luster y Rhoades, 1987; Morgan, Alwin y Griffin, 1979; Roberts, Block y Block, 1984; Schaefer y Edgerton, 1985; Segal, 1985; Wright y Wright, 1976).

La investigación sobre las expectativas de los padres en relación con sus hijos ha puesto en evidencia lo que acabamos de ver repecto al calendario evolutivo: mayor precocidad en los niveles sociales más elevados, así como previsión de niveles de desempeño más altos. Interés especial tiene, a nuestro juicio, la teoría clásica de Rodman sobre la dinámica de las relaciones entre valores y expectativas, teoría conocida como la de la elasticidad de los valores (Rodman, 1963; 
Rodman y Voydanoff, 1978); de acuerdo con las tesis de Rodman, las personas de status bajo comparten los valores dominantes de la sociedad en que viven, valores que suelen ser los de las clases altas y medias-altas; como quiera que tales personas encuentran difícil o imposible alcanzar esas metas, se ven obligadas a ampliar hacia abajo sus expectativas y aspiraciones, que teniendo su techo colocado a la misma altura que las personas de posición más favorecida, tienen su suelo mucho más abajo que ellas. El «efecto correctivo» de la realidad sobre las aspiraciones de los padres ha sido puesto de manifiesto en diversos trabajos revisados por Seginer (1983).

Uno de los ámbitos clásicos de estudio de las expectativas de los padres concierne a los aspectos diferenciales ligados al sexo. Aunque parece claro que con el paso del tiempo se va produciendo una atenuación de las expectativas estereotipadamente ligadas al sexo (Barnett, 1981; Intons-Peterson, 1985), persisten esterotipos que tienden a hacer variar las expectativas de los padres según que sus hijos sean niños o niñas y según además la faceta conductual de que se trate (Parsons, Adler y Kaczala, 1982).

Finalmente, hemos de referirnos a las actitudes de los padres, término por el que entendemos aquí su aceptación, rechazo o neutralidad respecto a una determinada faceta del desarrollo o la conducta de sus hijos. Como ya pusieron de manifiesto Newson y Newson (1963), los padres de nivel socioeconómico bajo suelen ser menos tolerantes respecto a aquellas conductas que discrepan de la norma o escapan a la conformidad, tendiendo a aumentar la tolerancia con el status social.

\subsection{Ideas sobre cómo aprenden los niños y cuál es el papel de los padres}

De acuerdo con McGillicuddy-DeLisi (1982b) y Sigel et al. (1980), las ideas sobre cómo aprenden los niños y cuál es el papel de los padres en el proceso de aprendizaje de sus hijos, varían en función de factores de constelación familiar (número de hijos y espaciamiento entre ellos), del status social de los padres y del sexo de padres e hijos. Así, por lo que a la constelación familiar se refiere, la principal diferencia resultó ser la mayor creencia en la enseñanza directa (transmisión de información a través de su presentación verbal) entre los padres de hijos únicos, en contraposición con la mayor insistencia en la autoregulación entre los padres de tres hijos. En relación con el status social, los padres de clase media se refirieron a los conceptos de feedback positivo, madurez, estructura del entorno, existencia de estadios evolutivos, etc., con mayor frecuencia que los padres de clase baja, que aludieron más frecuentemente a la enseñanza directa por parte de los adultos. Finalmente, en lo relativo a las diferencias en función del sexo de los progenitores, y en contraposición con las madres, los padres se refirieron con más frecuencia a inferencias extraídas de sus observaciones, a transmisión directa desde fuera y a madurez.

Según los mismos autores, los mencionados factores no actuarían en solitario, sino en interacción, dándose el caso de que los padres de clase media con hijos separados entre sí y los padres de clase trabajadora con hijos próximos entre sí se refieren con más frecuencia que los demás a procesos auto-reguladores.

De otro lado, diversos investigadores (Johnson y Martin, 1985; Ninio, 1979; Skinner, 1985; Tulkin y Cohler, 1973; Zukov, en este dossier) han abordado las diferencias en función del status social de los padres en el grado de influencia que éstos creen tener en el desarrollo de sus hijos. Sus datos coinciden en señalar que los padres de status alto perciben tener una mayor capacidad de influencia sobre el desarrollo de sus hijos, como corresponde a sus ideas más de corte inter- 
ccionista, mientras que los padres de status bajo, más proclives a ideas innatistas, presentan, consecuentemente, una percepción de capacidad de influencia más reducida.

Otros datos (Goodnow et al., 1986) han puesto de manifiesto que la capacidad de influencia sobre sus hijos que los padres perciben decrece a meciida que los niños se van haciendo mayores. Por otra parte, parece que la percepción de influencia es superior en unas áreas que en otras, pues, según Goodnow (1981), los padres se ven a sí mismos como más influyentes en el área social que en la cognitiva.

\subsection{Ideas sobre estrategias educativas}

El problema que en este terreno más ha atraído el interés de los investigadores ha sido el del control de la conducta de los niños por parte de los padres. Sin duda, el dato más consistente de los que se encuentran en la literatura tiene que ver con el carácter menos permisivo, más severo, de las estrategias educativas preferidas por los padres de status sociales más bajos, en contraste con las preferencias manifestadas por los padres de posiciones sociales más favorecidas, que se orientan en dirección a una mayor lenidad, o, en todo caso, a métodos de control conductual alternativos al castigo o las prohibiciones directas, lo que significa, a nuestro juicio, no que los padres de status alto ejerzan menos presión socializadora sobre sus hijos, sino que la ejercen de manera diferente (Goodnow et al., 1984; Lambert, Hamers y Frasure-Smith, 1979; Lautrey, 1980; Newson y Newson, 1963; McGillicuddy-DeLisi, 1982b; Sigel et al., 1980).

Antes de cerrar este apartado es preciso resaltar de nuevo el hecho de que las diferentes ideas de que hemos estado hablando están interconectadas entre sí, lo que no necesariamente significa que dependan unas de otras, pues un mismo padre puede sostener simultáneamente ideas contradictorias entre sí y además, como vimos, las ideas de los padres están probablemente agrupadas en subsistemas que no tienen por qué guardar entre sí una relación lógica. Según Applegate, Burke, Burleson y Delia (1985), entre los distintos índices del sistema de ideas existen correlaciones moderadas; la magnitud de tales correlaciones indica que existe una cierta coherencia entre los distintos ejes conceptuales de las ideas de los padres, pero sugiere también que estos ejes no son simples manifestaciones redundantes los unos de los otros.

\section{ORIGENES, DETERMINANTES Y FUENTES DE VARIACION DE LAS IDEAS DE LOS PADRES}

Las ideas de los padres sobre el desarrollo y la educación de sus hijos proceden de fuentes muy diversas y están sujetas a la determinación de muy distintas influencias; de unas y otras nos ocupamos a continuación separadamente, si bien los distintos determinantes de las ideas de los padres no ejercen su influencia por separado, sino conjunta e interactivamente.

Por lo demás, estamos de acuerdo con Hess y McDevitt (1986) en que los factores concretos implicados en la formación y posterior modificación de una idea específica no tienen por qué ser los mismos que los que están en el origen y posterior variación de una idea diferente.

\subsection{Transmisión cultural}

Los contenidos de buena parte de las ideas que en una cultura existen en torno al desarrollo y la educación de los niños están, en gran medida, «prefabri- 
cados» (Goodnow, 1985), son como un a priori con que el individuo se encuentra y que le suele resultar fácil asimilar. Como han señalado Sameroff y Feil (1985), algunos de estos contenidos son tan imperativos que resulta difícil sustraerse a ellos y adoptar puntos de vista diferentes.

Como han mostrado Le Vine (1974) y Whiting (1974), distintos grupos humanos que viven en circunstancias de adaptación muy diferentes modulan sus ideas y prácticas de crianza de manera tal que el resultado final sea máximamente adaptativo. En las sociedades tradicionales y en algunas ocasiones en nuestra cultura (Stevens, 1984), las ideas y prácticas de crianza se transmiten en el contexto en que son relevantes, sin necesidad de otros procedimientos. Sin embargo, entre quienes Whiting (1974) ha denominado alos neonómadas del mundo modernos, la ruptura de la familia extensa y las diferencias generacionales derivadas del cambio rápido en las condiciones de vida social, unido todo ello a la ausencia del trato asiduo con niños pequeños entre los adolescentes, se traducen, por una parte, en pérdida del peso otrora jugado por la tradición y, por otra, en que los padres jóvenes se enfrentan a sus responsabilidades más inermes de lo que estaban sus homólogos en sociedades tradicionales.

El grupo cultural a que se pertenece aporta no sólo un marco ideológico general que en mayor o menor grado se transmite de una generación a otra. El contexto cultural contiene también una serie de estereotipos específicos relativos a muy diversas cuestiones, que funcionan como auténticos a priori específicos y que influyen sobre la forma en que se perciben determinados fragmentos de la realidad (ver, por ejemplo, Bryan, Coleman, Ganong y Bryan, 1986).

\subsection{La propia experiencia como hijos}

Aunque parece intuitivamente razonable postular que una de las fuentes de las ideas que nos ocupan radica en las propias experiencias infantiles (en los recuerdos que los padres guardan de cómo fue su propia crianza, cuáles fueron las actitudes y prácticas de sus propios padres, etc.), las evidencias empíricas son contradictorias. Por un lado, cuando a los padres se les pregunta de dónde creen que proceden sus ideas evolutivo-educativas, tienden a responder en elevadas proporciones que de su propia experiencia como hijos, según la menos los datos de Sigel et al. (1980). Pero, por otro, cuando se evalúan las ideas de los padres y las de sus hijos, unas y otras tienden a coincidir muy poco, de acuerdo ahora con los datos de Cashmore y Goodnow (1985) y de Russell y Rusell (1982).

\subsection{Ia propia experiencia como padres}

También en el caso del papel que la propia experiencia como padres juega como moldeadora de las ideas evolutivo-educativas, las evidencias son contradictorias. Están, de una parte, aquellos autores que, como Holloway y Hess (1985), McGillicuddy-DeLisi (1982a y b), Sigel et al. (1980) y Stevens (1984), encuentran datos que hablan claramente a favor del hecho según el cual el contacto y la experiencia con los propios hijos no sólo están en el origen de las ideas de los padres, sino que son también el motor de su variación. De otra parte están los autores que, como Alwin (1984), Brooks-Gunn (1985), Goodnow (1985) y Ninio (1979), no encuentran que, en general, la experiencia como padres sirviera para cambiar las ideas evolutivo-educativas sostenidas previamente.

Goodnow (1985) ha sugerido que tal vez la clave no radique tanto en la experiencia con niños, cuanto en la capacidad de ssintonía finas que con ellos se tenga y de la que derivarán en mayor o menor medida datos que harán cambiar más o menos las ideas previas. 


\subsection{El status socioeconómico}

Es siempre difícil decidirse respecto a cómo definir y utilizar las variables asociadas con el status: El nivel profesional, el de ingresos, el de escolarización, etc., se utilizan a veces de forma indiscriminada para definir el status. Es cierto que los diferentes índices posibles están asociados entre sí y son con frecuencia redundantes, lo que no facilita una consideración separada del efecto de unos y otros. Hablaremos después del papel jugado por el nivel de escolarización, refiriéndonos aquí a la influencia de los demás indicadores.

Uno de los puntos de vista más clásicos sobre estas cuestiones es el ya aludido de Kohn (1969, 1976, 1977; Kohn y Schooler, 1969), según el cual hay ocupaciones profesionales que promueven los valores de auto-dirección y otras que dan pie a valores relacionados con el conformismo. En realidad, valoran la autodirección quienes la experimentan de hecho en su actividad profesional, mientras que el conformismo procede de ocupaciones que lo promueven, antes que a nivel de los valores, a nivel de la adaptación cotidiana. Mientras que hay autores cuyas investigaciones no confirman las tesis de Kohn (por ejemplo, Burns et al., 1984), en otros casos la confirmación sí ocurre (por ejemplo, Ispa, Gray y Thornburg, 1984; Luster y Rhoades, 1987; Morgan, Alwin y Griffin, 1979).

El caso de Lautrey (1980) es distinto, pues aunque los datos de su investigación son similares a los de Kohn, él sugiere interpretaciones alternativas. Para Lautrey, como también para Newson y Newson (1963), son las condiciones de vida las que determinan el universo de valores de los padres; así, la limpieza, el orden y è cuidado de las cosas son importantes cuando los padres no disponen de posibilidades económicas para reemplazar lo manchado, lo perdido o lo roto; las restricciones y la obediencia son importantes cuando la falta de espacio no permite mucha libertad de acción.

\subsection{El nivel educativo}

Son numerosísimos los autores que han encontrado en sus investigaciones cómo el nivel educativo de los padres es el predictor más potente de sus ideas sobre el desarrollo y la educación de sus hijos (entre otros, Alwin, 1984; Boltanski, 1969; Bronfenbrenner, Alvarez y Henderson, 1984; Laosa, 1982a y b; Wright y Wright, 1976). Existe una cierta coincidencia en afirmar que la prolongación de la escolarización transforma el babitus del sujeto, le incita a adquirir conocimientos que la escuela no transmite, le da más capacidad de análisis y de crítica.

Aunque el papel del nivel de estudios sea considerado por muchos el determinante principal de las ideas evolutivo-educativas de los padres, la concordancia que frecuentemente se da en las mismas personas entre el nivel educativo y otros índices de status (nivel de ingresos, profesión, modo de vida...), hace que, de hecho, la influencia de estas variables asociadas sea acumulativa y potencie mancomunadamente uno u otro tipo de ideas.

\subsection{Diferencias individuales}

Además de los factores generales a que hemos estado refiriéndonos en este apartado, deben considerarse como influyentes sobre el tema que nos ocupa los factores diferenciales. En este sentido, las ideas evolutivo-educativas de los padres se ven influenciadas por características de los niños tales como su edad, su sexo, su temperamento, su responsividad, sus capacidades (Dix y Grusec, 1985; Dix, Ruble, Grusec y Nixon, 1986; Hess et al., 1980; Jacob, Fagin, Perry y Van 
Dyke, 1975; Lamb y Easterbrooks, 1981; Roberts, Block y Block, 1984; Sameroff, 1975).

Pero si las características de los niños juegan un papel importante, otro tanto, como mínimo, puede decirse respecto a las de los padres. Su inteligencia, su estilo congnitivo, sus características de personalidad, su edad, su sexo, introducirán sin duda elementos diferenciadores cruciales (Bacon y Asomore, 1985. 1986; Bates y Bayles, 1984; Goodnow, 1985; Lautrey, 1980; McBride, 1985; Newson y Newson, 1963; Pharis y Manosevitz, 1980; Sameroff y Feil, 1985; Schaefer y Edgerton, 1985; Sigel, 1985c; Wegner y Vallacher, 1977).

Finalmente, las ideas de los padres varían no sólo en función de sus propias características y de las de sus hijos, sino también en función de las relaciones que existan entre unos y otros, como han mostrado Hess y McDevitt (1986) a propósito de las relaciones afectivas entre un grupo de madres y sus hijos. A la modulación del sujeto sobre las ideas se ha referido también Sigel (1986).

Hay un aspecto en el que lo interesante es preguntarse por el grado de semejanza o discordancia que exista entre las ideas de unas y otras personas. Nos referimos al mayor o menor grado de acuerdo existente entre las ideas de los dos miembros de un matrimonio. Es éste un dato sobre el que diferentes autores han encontrado realidades distintas, pues mientras algunos han hallado convergencias importantes entre marido y mujer (Bronfenbrenner et al., 1984; Triana y Rodrigo, 1985), otros han hallado como predominantes las discordancias (Bacon y Ashmore, 1985, 1986; Bates y Bayles, 1984).

\section{DE LAS IDEAS DE LOS PADRES A SUS CONDUCTAS CON LOS HJOS}

No es posible en este apretado resumen compendiar la ya voluminosa literatura producida en torno a la relación ideas de los padres-conductas interactivasdesarrollo y desempeño del niño. La idea de fondo de quienes se han ocupado del análisis de dicha relación es la de que los comportamientos de los padres con sus hijos están mediados por sus ideas evolutivo-educativas (Bacon y Ashmore, 1986; McGillicuddy-DeLisi, 1982a y b; McGillicuddy-DeLisi et al., 1979; Sigel, 1986; Sigel et al., 1980; Sameroff y Seifer, 1983) y lo que las investigaciones empíricas han tratado de mostrar ha sido que en efecto tal mediación es un hecho.

Han sido varias, no obstante, las investigaciones en las que no se han encontrado relaciones significativas -o las encontradas han sido muy débiles-entre las medidas de ideas, las de conducta y las de desarrollo o desempeño de los niños (Hess et al., 1980; Moran y O'Brien, 1984; Newson y Newson, 1963; Roosa, 1984; Skinner, 1985; Turner y Harris, 1984).

Sin embargo, las investigaciones que sí han hallado significativas las mencionadas relaciones, son mucho más numerosas. Tal es el caso en la relación ideas de los padres-interacciones tempranas con sus hijos (Broussard y Hartner, 1971; Engfer y Gavranidou, 1984; DeVries y Sameroff,. 1984; Donovan, Leavitt y Balling, 1978; Hess, 1981; Milliones, 1978; Spangler, 1986; Stern y Hildebrandt, 1986), en la relación ideas de los padres-desempeño intelectual y rendimiento académico del niño (Brooks-Gunn, 1985; Holloway y Hess, 1985; Hunt y Paraskevopoulos, 1980; Lautrey, 1980; McGillicuddy-DeLisi, 1985; Miller, 1986; Price y Gillingham, 1985; Schaefer y Edgerton, 1985; Seginer, 1983; Sigel et al., 1980) y en la relación ideas de los padres-prácticas de socialización (Brooks-Gunn, 1985; Clarke-Stewart, 1973; Dix y Grusec; 1985; Fry, 1985; Mancuso y Handin, 1985; Palkovitz, 1984; Segal, 1985; Stevens, 1984; Tulkin y Cohler, 1973). 
más de manifiesto que, aunque frecuentemente se produzca, la conexión ideasconducta no está sujeta a una relación mecánica y lineal tal que conocidas las ideas se pueda hacer una predicción ajustada de la conducta que de ellas emanará. En este sentido se han pronunciado entre otros muchos Elias y Ubriaco (1986), Fishbein (1980), McGillicuddy-DeLisi (1982b), Rokeach (1980) y Sigel (1986). Este último autor se ha servido de la ametáfora del embudo» (Sigel, 1986, p. 57) para expresar la idea según la cual si bien es claro que no existe una correspondencia punto por punto entre ideas y conducta, puede ocurrir que la determinación de una acción venga dada no por una idea aislada, sino por la mezcla de varias; es como si en un embudo se vertiera un cierto número de ideas, siendo luego el resultado un número reducido de acciones. Debe quedar claro, no obstante, que lo que se vierte en el embudo no son sólo ideas, sino también otros determinantes (del contexto, de la tarea, de los sujetos...) que interactúan unos con otros y también con las ideas.

En todo caso, la forma en que las ideas de los padres hallen materialización en su conducta dependerá, más bien, de una serie de factores moduladores que determinarán en última instancia, y a partir de dichas ideas, el curso de acción que se seguirá con más probabilidad. Entre esos factores moduladores los hay relacionados con los padres y sus características, con el niño y las suyas, y, finalmente, con la situación en la que unos y otros se encuentran e interactúan. Algunos de los autores que se han ocupado de estas cuestiones son Brooks-Gunn (1985), Goodnow et al. (1986), Kuczinsky (1984), McGillicuddy-DeLisi (1982b), Palkovitz (1984) y Sameroff y Feil (1985).

Finalmente, es preciso mencionar que las ideas de los padres encuentran traducción no sólo en conductas específicas con sus hijos, sino también en la forma en que organizan y estructuran el entorno familiar en relación con el niño (horarios, hábitos, objetos, etc.) (Lautrey, 1980; McGillicuddy-DeLisi, 1985; Parks y Smerliglio, 1986; Seginer, 1983).

\section{UN MARCO TEORICO PARA LOS DATOS}

Buena parte de los datos que hasta aquí hemos venido comentando se compendian en la Figura 1, de la página siguiente, respecto a la cual es preciso hacer dos breves comentarios. El primero, con relación al cuadro que contiene las ideas de los padres; como se ve, las ideas evolutivo-educativas de carácter general están unidas por una flecha a las ideas ligadas a la acción, con lo que se indica que éstas derivan de aquéllas, pero están unidas también por una línea partida que indica que las ideas-acción no necesariamente emanan de las generales. El segundo comentario se refiere a los factores que influyen sobre el nivel de desempeño y desarrollo del niño; entre ellos están, como se ve en la Figura 1, las influencias educativas extrafamiliares, en las cuales las ideas y prácticas educativas de los profesores parecen jugar un papel importante (Bauch, 1984; Clark y Peterson, 1986; Munby, 1982; Reighart, 1984; Rogers, 1982; Rosenthal y Jacobson, 1968; Shavelson, 1983; Tachachnick y Zeichner, 1985; Yinger, 1986).

$\mathrm{Si}$ los datos acumulados por los investigadores tienen una cierta coherencia y permiten su integración en una visión de conjunto como la de la Figura 1, resulta del máximo interés encuadrarlos en un marco teórico que permita transcender la mera constatación de hechos y nos aproxime a una conceptualización general del papel que las ideas evolutivo-educativas de los padres juegan en el desarrollo de sus hijos y de cómo se ejerce tal influencia. Entre otras cosas, porque las aproximaciones a la temática que nos ocupa se suelen caracterizar por ser marcadamente ateóricas y por carecer, por tanto, de un encuadre conceptual 
que dé sentido a los datos más allá de las hipótesis específicas y las interpretaciones ad hoc.

FIgURA 1

Las ideas de los padres: origenes, dinämica y consecuencias.

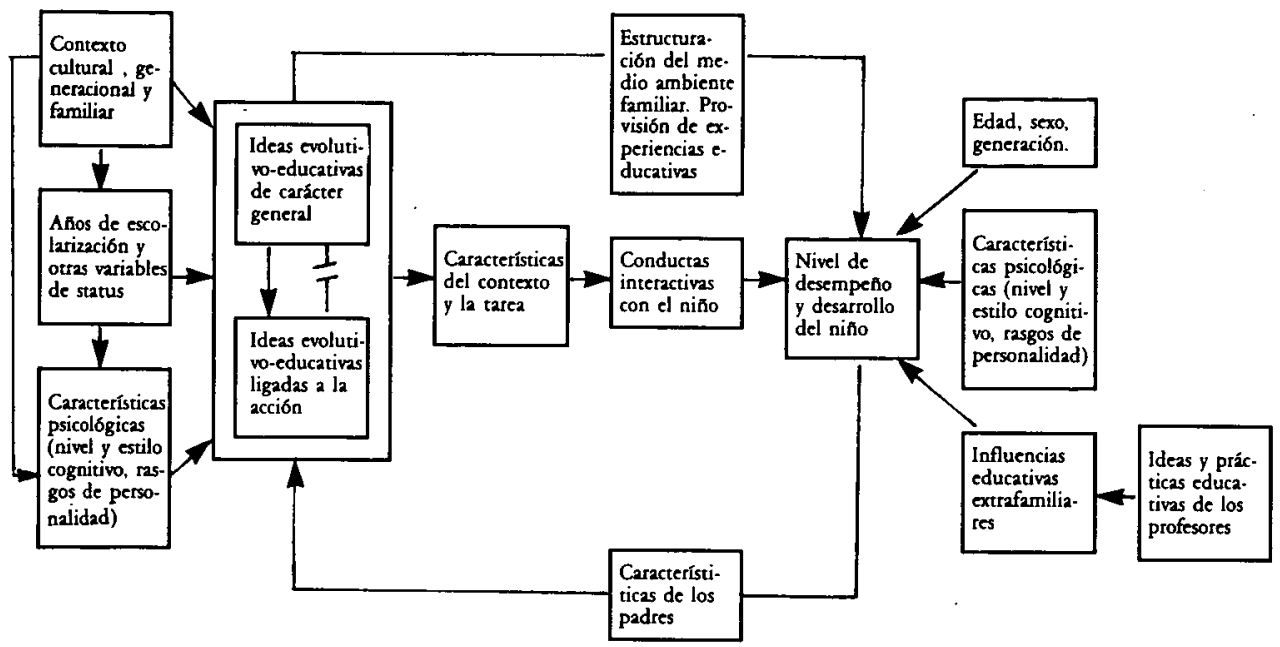

Por nuestra parte, creemos que las ideas de Vygotski (1934/1984; 1935/1979) proporcionan materiales conceptuales con los que construir un marco en el que los datos se encuadren y adquieran significado. En otro lugar (Palacios, 1987) hemos propuesto, a este respecto, aprovechar la noción de zona de desarrollo próximo (ZDP) y hemos resaltado cómo, por su propia definición, esta noción implica por un lado al niño y por otro al adulto con el que interactúa. Porque lo que es crucial en el concepto de ZDP es que no se trata de algo que exista ya preformado dentro del niño, sino de una posibilidad que requiere la interacción adulto-niño para hacerse realidad. La ZDP es una posibilidad existente en el niño, aunque no una posibilidad sin límites, dado que desde su nivel de desarrollo actual no le es posible acceder a cualquier desarrollo potencial, sino sólo a aquellos que estén al alcance de sus posibilidades evolutivas de cada momento. Pero puesto que muchos de estos desarrollos requieren, para producirse, asistencia y guía, es necesario que el adulto perciba en el niño la posibilidad y encuentre la forma de traducir en acciones concretas el apoyo que el niño necesita para alcanzarla. Así, las posibilidades de desarrollo existentes en el niño (la zona de desarrollo a la que puede acceder con ayuda) requieren para hecerse efectivas que el adulto las perciba (algo así como una «zona de desarrollo próximo percibido») y actúe luego con apoyos eficaces.

Cuando adulto y niño interactúan lo hacen a propósito de un tema, de una tarea, interactúan en una situación. Esta interacción adulto-niño implica, tanto por parte del adulto como por parte del niño, una cierta «definición» o «representación de la situación» (Wertsch, 1984), pero implica también, por parte del adulto, una cierta definición o representación de lo que es un niño, de cómo se desarrolla, de en qué medida y de qué manera es posible ayudarle a conseguir nuevos logros. Tal definición o representación puede entenderse como dotada de al menos dos vertientes: una general, referida al desarrollo y la educación en términos abstractos (cómo se desarrollan los niños, cómo aprenden, cómo se les puede ayudar, etc.), y otra específica, referida a un niño concreto y a una situación o contexto interactivo determinado (cómo se ha desarrollado este niño, cómo aprende, cómo se le puede ayudar en esta situación a lograr este objetivo, etc.). 
El concepto de ZDP es profundamente interpersonal y, por definición, el tránsito asistido de los potencial a lo real requiere de alguien más capaz que percilo real y se sienta capaz de actuar sobre ello. Aquí es donde a nuestro entender encajan las percepciones de los padres, sus ideas, sus expectativas sobre calendarios y logros evolutivos, el grado de influencia que creen tener sobre sus hijos, etc. En todos estos aspectos, el desarrollo que los padres perciben como posible puede coincidir en gran medida o escasamente con el potencial de desarrollo que su hijo realmente tiene. En la medida en que uno y otro aspecto no son siempre, con toda probabilidad, superponibles e idénticos, preferimos decir que la zona de desarrollo próximo tiene dos vertientes de igual importancia y que deben ser igualmente enfatizadas: la posibilidad de desarrollo que hay en el niño y la percepción de esa ponibilidad por parte de quienes interactúan con él.

Se puede formular la hipótesis de que cuanto más amplia sea esa «zona de desarrollo próximo percibido», es decir, cuanto más ajustadas y optimistas sean por parte de los padres las previsiones de desarrollo posible en el niño, tanto más probable será que den lugar a interacciones que, iniciadas y controladas por los adultos, y de acuerdo con la ley del origen social de las funciones psíquicas, acaben por convertirse en desarrollo.

De acuerdo con esta propuesta, las ideas de los padres sobre el desarrollo y la educación de sus hijos determinan la zona de desarrollo próximo percibido, es decir, la zona de desarrollo que los padres intuyen, averiguan, esperan y ensayan en sus hijos; su conducta con el niño se verá influida por esa percepción y acabará dando lugar a desarrollo a través del eandamiajes con el que los padres construyen el desarrollo conductual transformando en real la zona de desatrollo potencial.

Esta conceptualización permite entender por qué, según el punto de vista de Bugental y Shennum (1984), que suscribimos, el educador eficaz es, desde una perspectiva transaccional, el que tiene un conjunto de ideas y realiza un conjunto de actividades que sirven para mantener a la vez la eficacia personal propia y la del niño al que se educa. Al fin y al cabo, la zona de desarrollo próximo percibido está definiendo no sólo lo que los padres piensan que el niño puede lograr, sino también los límites de su capacidad para ayudarle a hacer efectivos esos logros.

\section{Resumen}

Este articulo presenta una revisión de literatura sobre el tema de las ideas de los padres sobre el desarrollo y la educación de sus bijos. Se analizan los antecedentes históricos y posteriormente se discuten la diversidad, los contenidos y los determinantes de las ideas evolutivo-educativas de los padres. Después de una referencia a las relaciones ideas-conducta-desarrollo, se propone para este ámbito de investigación un marco teórico elaborado a partir de las propuestas de Vygotski.

\section{Summary}

In this paper, literature about the topic of parent's ideas about children's development and education is reviewed. After analyzing their bistorical antecedents, the diversity, contents and determinants of parental ideas about development and education are discused. After a reference to the connection ideas-behavior-development, a theoretical frame is proposed, frame elaborated according to Vygotski's conceptual proposals. 